\title{
A Conservative Management of Keratocyst Odentogenic Tumor Using Cornoy's Solution And Osteoinduction using Concentrated Growth Factor Mixed with Aotogenous Bone Graft - Randomized Clinical Research.
}

\author{
V.Sadesh Kannan ${ }^{1}$, E.Elavarasi ${ }^{2}$, M.Mahalakshmi ${ }^{3}$, K.Vinod Krishna ${ }^{4}$, \\ R.Muralidaran ${ }^{5}$, Prithivi Sankar Nanthakumar ${ }^{6}$. \\ ${ }^{I}$ (Professor \& Head, Department Of Dentistry, Arupadaiveedu Medical College \& Hospital, Vinayaga Mission \\ University, Puducherry, India) \\ 2,3,4,5,6 (Department Of Oral \& Maxillofacial Radiology \& Surgery, Adiparasakthi Dental College \& Hospital, \\ Tamilnadu Dr.Mgr Medical University, Melmaruvathur)
}

\begin{abstract}
:
Aim: The objective of this study is the complete elimination of the Keratocystic Odentogenic Tumor (KCOT) along with lining epithelium at the initial operation and induces osteoblastic activity using Concentrated Growth Factor (CGF) derived from autologus blood.

Materials: 10 cases were involved in the study; Male: Female ratio 6:4; Maxilla: Mandible ratio 5:5, with a mean age of 36 years.

Method: All cases of KCOT were treated by enucleation and chemical cauterization using cornoy's solution followed by placement of CGF in the dead space along with aotogenous bone graft mixed with red blood corpuscular layer.

Results: Results were excellent in all the patients with very good ossification at the operated site and none of our patient had recurrence or post-operative complication.

Conclusion: This conservative management of KCOT using cornoy's solution and placement of CGF allows excellent ossification of new bone which in turn useful for future rehabilitation.

Keywords: Keratocystic Odentogenic Tumor;Odentogenic Keratocyst;Cornoy's solution;Concentrated Growth Factor, Aotogenous Bone Graft.
\end{abstract}

\section{Introduction}

Since Philipsen in 1956 introduced the term odentogenic keratocyst (OKC) to describe cyst of the jaws lined by keratinizing epithelium, for the long postulates clinicians have different school of thought with a rational approach to the treatment. WHO has changed the term OKC as Keratocystic Odentogenic Tumor (KCOT), the serious problem is that these cysts have high recurrence following removal and if untreated may lead to further bone destruction and soft tissue involvement ${ }^{1}$. The possible mechanism for the recurrence of OKC were described by Voorsmit et al in 1981, stated that any cystic epithelium left behind in the cavity may give rise to new cyst formation from the daughter cyst, micro cyst or epithelial island can be found in the wall of the original cyst ${ }^{2}$. Our objective is the complete elimination of the lesion along with lining epithelium at the initial operation and induces osteoblastic activity using Concentrated Growth Factor (CGF) derived from patients own blood.

Growth Factors were first introduced by Choukroun, states that Proteins which help in wound healing has main role in cell migration, cell proliferation and angiogenesis in wound healing, completely free from any viral diseases; CGF is an innovative growth factor for producing a fibrin matrix with aotogenous bone graft ${ }^{3}$. SACCO In 2006 developed CGF, which is produced by centrifuging blood samples with a digital centrifuge device ${ }^{4}$.

\section{Materials}

In this research 10 samples were involved; Male: Female ratio 6:4; Maxilla: Mandible ratio 5:5, with a mean age of 36 years, all cases proved as OKC histopathologically by incisional biopsy were involved in the study. Exclusive criteria ASA-II, \& ASA-III, patient with Anemia, systemic disease like uncontrolled diabetes, Haemophilia, aoto-immune disorder etc, malignancy like Leukaemia were excluded from the study. In this study, two different tubes containing intravenous blood sample of the patient, were centrifuged with a CGF Centrifuge (MEDIFUGE ${ }^{\mathrm{TM}}$, Silfradentsrl, S. Sofia, Italy) 


\section{Methods}

General Anaesthesia was administrated using nasal intubation of endo-tracheal tube. Buccal or labial vestibular incision were placed (Fig\# 1), muco-periosteal flap were raised and buccal corticotomy was done through the breach of the cyst or thin buccal cortex. Enucleation of the KCOT along with its lining epithelium in Toto using bone curette (Fig\#2) and specimen was send it for histopathological report (Fig\#3). Chemical cauterization of the cystic cavity was done using Cornoy's solution (Absolute alcohol- $5 \mathrm{ml}$; Chloroform- $3 \mathrm{ml}$; Glacial acetic acid- $1 \mathrm{ml}$; \& Ferric chloride- 1 in 1000 parts) socked gauze for 5 minutes (Fig\#4), after preserving the peri oral soft tissue using Vaseline gauze or sofratule gauze to prevent chemical burn. After removing the gauze, the blackish decolourization of left cystic lining (Daughter cyst or satellite cyst) due to chemical cauterization were removed using bone curette (Fig\# 5), and irrigation of the cavity using normal saline.

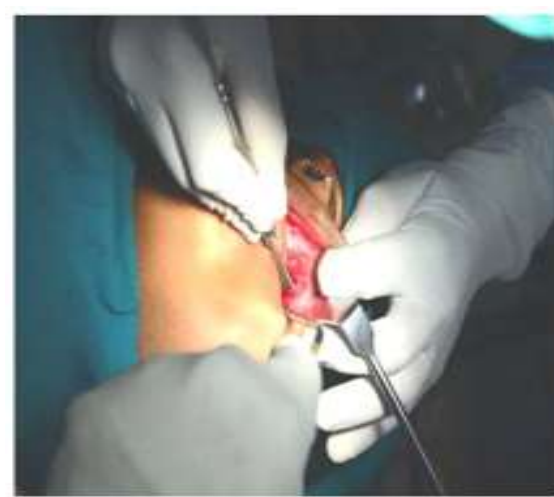

Fig $=1$ :Intra-Oral Buccal Vestibular

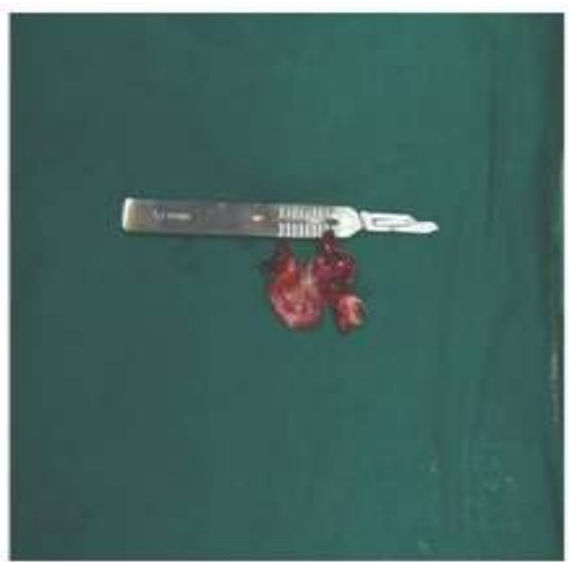

Fig=3: Histopathological Specimen

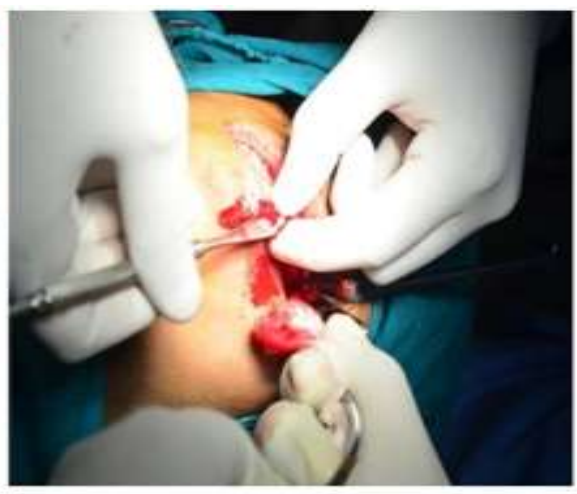

Fig $=3$ : Enucleation OfCystic Lining

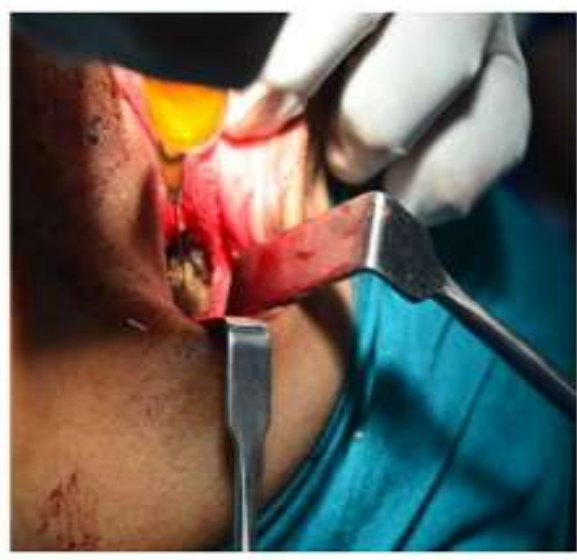

Fig-4: Iniecting Camov's Solution

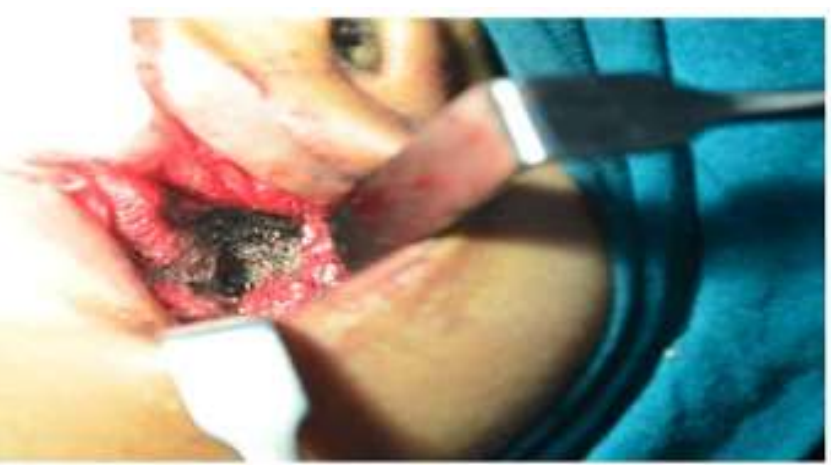

Fig\#5: Cemicaly Cauterized Cystic Walls 


\section{Preparation of CGF:}

$9 \mathrm{ml}$ autologous blood was drawned from the patient and centrifuged at 30 " acceleration at the rate of 2700 RPM and 36" deceleration with 3600 RPM and stopped (Fig\#6).Three layer of prepration was formed, Upper a cellular plasma, middle fibrin plug containing concentrated growth factor and lower Red Blood corpuscular Layer was found (Fig\#7). The upper a cellular plasma was discarded and the middle fibrin plug containing CGF was cut along with some $\mathrm{RBC}^{5}$. (Fig\# 8)

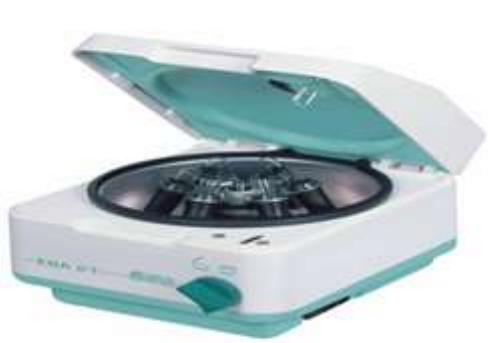

FIG\#6: CENTRIFU GE (MEDIFUGE TM, Silfradentsti. S. Sofia, Italy)

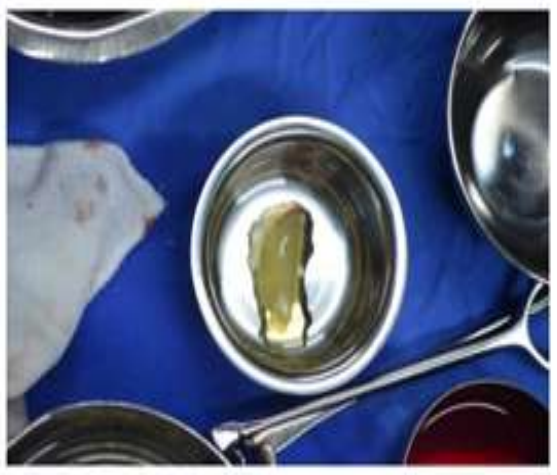

FIG\#8: FIBRIN PLUG RICH CGF CENTER LAYER
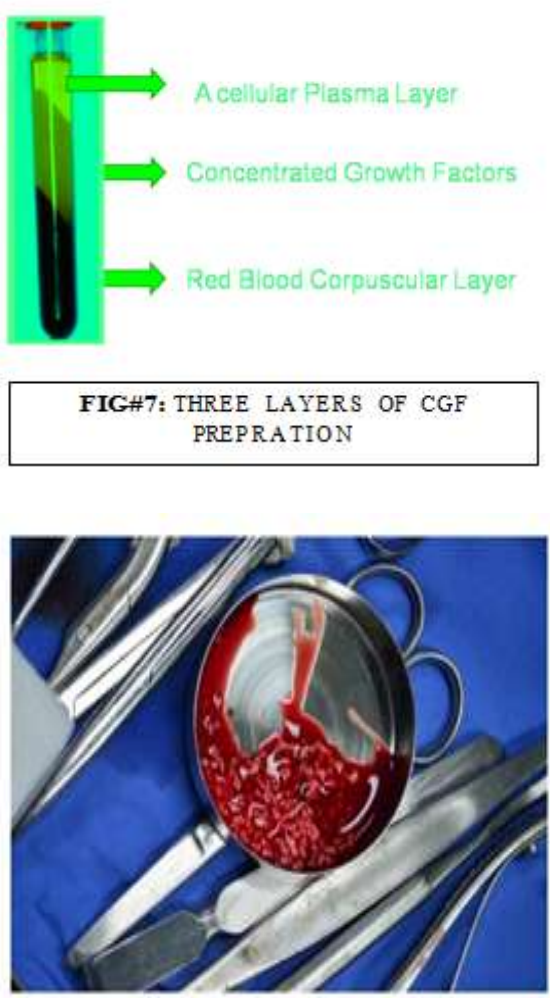

FIG\#9: AUTOGRAFT MIXED WITH REMAINING RBC

\section{Autogenous Bone graft:}

Bone graft for maxillary lesion was taken from mandibular symphysis region and for mandibular lesion, the donor site is maxillary antral anterio-medial wall. After harvesting, the graft was crushed in to pieces and mixed with remaining RBC in semi-solid form (Fig\#9). and placed in the dead space cavity along with CGF and water tight closure was done using 3-0 Vicryl

Post-operative Intravenous Anti-biotic were given for five days, and wound care were done using intraoral irrigation with normal saline and betadine. All patients were evaluated clinically for wound dehiscence, wound gapping, pain (VAS), and swelling, ossification was evaluated using Orthopantamogram (OPG) preoperatively (Fig\#10, \& 12), post-operatively $1^{\text {st }}$ week, $1^{\text {st }}$ month, $3^{\text {rd }}$ month (Fig\#11, \& 13$), 6^{\text {th }}$ month, 1 year.

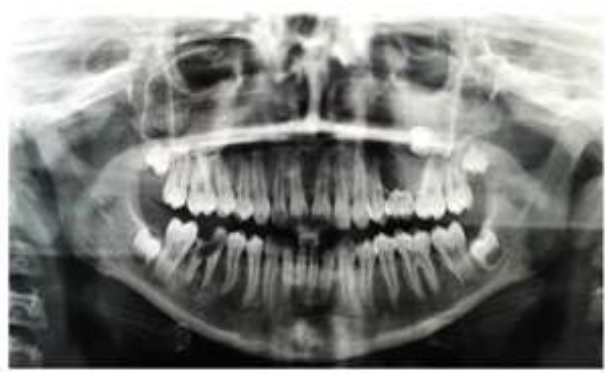

FIG\#10: PRE-OP OPG OF CASE: 1- OKC IN MAXILLARY ANTRUM

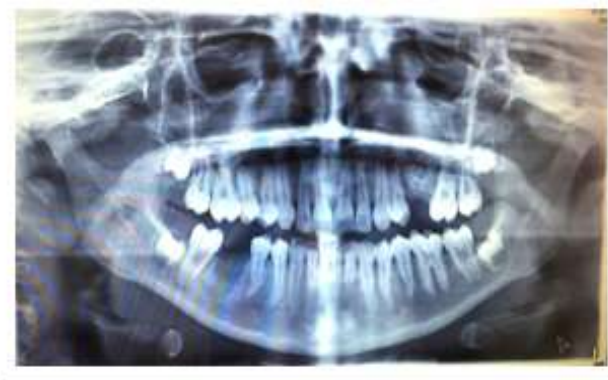

FIG\#12: POST-OP OPG OF CASE: 1 - OKC IN MAXILLARY ANTRUM 

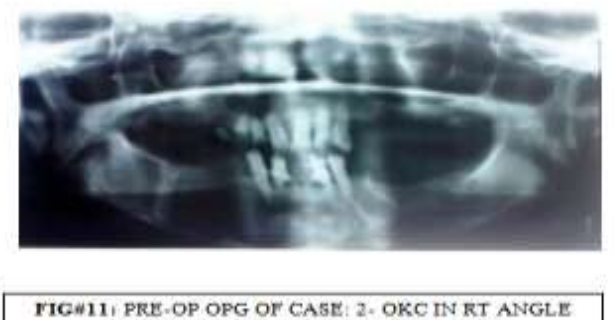
OF MANDIBLE TO LT CANINE REOION

IV.

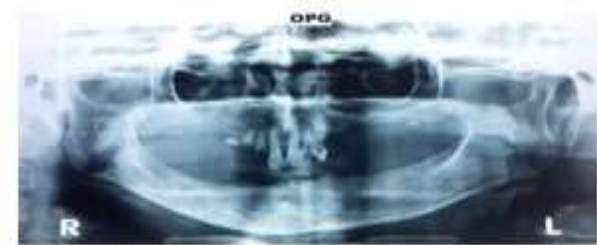

FIGN13, POST-OP OPG OF CASE: 2-OKC N LT ANGLE OF MANDIBLE TO RT CANINE REGION.

Results

Two of our patient was followed up for more than 2 years, Three patients more than 1 year, Three were more than 6months and two of our patients were followed just for more than 3 months, and we concluded this result. Post-operative results were excellent in all the patients with very good ossification at the operated site were well evident in OPG on the $3^{\text {rd }}$ month, and none of our patient had recurrence or post-operative complications. Post-operative pain was present for two patients in the adjacent tooth, which was well managed by root canal treatment, all of our patient had diffuse swelling following surgery, which is rather than a complication it is a normal post-operative sequel or oedema, which were managed by 1 stat of $8 \mathrm{mg}$ intravenous injection of dexamethasone post-operatively.

\section{Discussion}

Anyhow the presence of an Keratocystic odentogenic tumor can be provisionally diagnosed on clinical or radiographic grounds, histopathological confirmation is required for the confirmative diagnosis. Complete removal of keratocyst in one piece is often difficult because of thin and fragile bony wall of the cyst and hence the recurrence rate is high.

Bramley in 1971, suggested a rational surgical approach, he advocated that the small single lesion should be completely enucleated along with the mucoperiosteum overlying, while the large single lesion in the mandible should be similarly treated, if necessary through the extra-oral approach. He recommended that the multiloculated lesion should be operated by marginal resection along with the overlying mucosa, and the multicystic lesion, if small, in a similar manner, larger lesion is managed by resection and bone grafting ${ }^{6}$. This rational approach is supported by Shear (1983). Others however, have advocated marsupialisation, a method Shear does not favour because he consider that proliferating dental lamina and developing satellite cyst may occur in the fibrous wall of the primary cyst cavity ${ }^{7}$.

Various method has been introduced to evacuate the residual cyst epithelium from the bony cavity after enucleation. Bradly and Fisher in 1975 proposed removal of overlying mucoperiosteum, and enucleation of the cystic lining using cryosurgery through any small bony perforation followed by freezing of entire cavity. This is achieved with liquid nitrogen spray, the duration of freezing determined by thermocouples placed in the outer cortex of the mandible whilst freezing the entire mandible, so that a minimum of $20^{\circ} \mathrm{C}$ temperature is measured ${ }^{8}$.

In selected cases of large OKC's marsupialisation to permit cyst shrinkage, followed by enucleation, may be an alternative method.

Many surgeons recommend peripheral osteotomy of the bony cavity using a bone bur to minimise the intensity of recurrence. Others suggested, after cystotomy and incisional biopsy, some surgeons have treated large KCOTs by insertion of polyethylene drainage tube to allow decompression and subsequent reduction in size of the cystic cavity. Such decompression treatment results in thickening of the cyst lining, allowing easier removal with an apparently lower recurrence rate.

Fixing agents have also been used for irrigating the bony cavity following cyst removal. Carnoy's solution was first recommended by Voorsmit et al in 1981. They reported that this method of treatment is followed by a $2.5 \%$ recurrence rate compared to a $13.5 \%$ recurrence rate in untreated cases ${ }^{2}$.

Carnoy's solution is used for chemical cauterization of the bony cavity after cyst removal. Intra - luminal injection of carnoy's solution also has been used to separate the cyst from the bony wall, which make easy removal of entire cystic lining with a lower recurrence rate ${ }^{2}$.

There is a desire and need to be able to reconstruct the portion of lost or damaged tissue is always been one of the most extensively studied therapeutic aspect of regenerative medicine Guided Tissue Regeneration (GTR) - has gone through different iterations using different types of natural and synthetic materials to construct membrane and cavity fillers Eg : Goretex, Pericardium, Hydroxy Apatite, Tricalcium Phosphate, Poly Glycolic acid, Animal/ Human Bone Though these have shown various success, they are not alive ${ }^{9}$. CGF was first developed by Sacco. CGF is produced by the centrifuge of the venous blood as like in PRF. But, the technique differs by the centrifugation speed. Unlike PRF, CGF uses variable 
speeds from 2400 to $2700 \mathrm{rpm}$ to separate cells in the venous blood. This results in fibrinrich blocks that are much larger, denser, and richer in growth factors than PRF, The growth factor present in fibrin plugs are Bone Morphogenic Proteins (BMP); Fibroblast Growth Factor (FGF); Insulinlike growth Factor (IGF); Platelet Derived Growth Factor (PDGF); Transforming Growth Factor - Beta (TGF Beta); Vascular Endothelial Growth Factor (VEGF), which in turn results in a better regenerative capacity and greater versatility when using the fibrin-rich block. At $3000 \mathrm{rpm}$ for $12 \mathrm{~min}$. Centrifugation divided the blood into four layers, and the second layer or buffy coat and the third layer, also called growth factor layer, were made up of the CGF. PRP and PRF are $1^{\text {st }}$ $\& 2^{\text {nd }}$ generation fibrin rich block but CGF is the present $3^{\text {rd }}$ generation fibrin rich growth factor. Based on these results, the use of PRP, PRF, and CGF facilitates osteoinduction in the early stage of bone graft healing. After the 12th postoperative week, there was no difference in osteoinduction among the growth factors. So that, the authors consider that further studies about the long-term effects of PRP, PRF, and CGF must be done ${ }^{5}$.

CGF comprises an autologous fibrin matrix that has intrinsically incorporated a large quantity of plasmatic and leukocytic cytokines. The coagulation of fibrin plays a very important mechanical role thanks to its rigidity and mixed with the graft materials, it forms a biological binder. Once the CGF and the graft materials have been placed at the required site it induces the migration of endothelial cells, neutrophil migration and increase the capacity of receptors CD 11 and CD 18 on the surface and helps in the process of neoangiogenesis. The plasmatic cytokines help facilitate regular healing of the wound. The immune aspect is very important, due to presence of leukocytes in the coagulation.

\section{Summary}

Other than the tendency for recurrence, the overall prognosis for most Keratocystic Odentogenic tumor is impressive. But rarely, a locally aggressive Keratocystic odentogenic tumor cannot be controlled without local resection and bone grafting. In extremely rare instances, keratocyst have been seen to extend up into the small base region. Carcinoma arising from the Keratocystic odentogenic tumor has been reported, but the propensity for a KCOT to undergo malignant transformation is no greater and is possibly less than that for other types of odentogenic cysts. Patients with KCOTs should be evaluated for manifestations of the nevoid basal cell carcinoma syndrome

We follow up our cases for 6 months and no signs (or) evidence of recurrence were found and post operative OPG was taken to see the progress and osteogenesis. We faced no major complications.

The advantages of CGF are Inexpensive, Easy to prepare, No biochemical manipulation of blood, No potential risks associated the use of bovine thrombin, excellent osteoinduction.

\section{Conclusion}

The advantages of this technique are that the need for rational approach may be obviated and it is relatively simple to either intra - oral (or) external approach to extensive cyst. In this way the maintenance of mandibular continuity and maxillary antral anatomy and esthetics is achieved, thus preserving jaw function and shape. Rapid healing is promoted, secondary infection is decreased by obliterating the dead space using CGF mixed with bone graft; and the inferior dental neurovascular bundle can be preserved. This method shortens the operating time, thus reduces the donor site morbidity.

In these patients, it is essential that close follow up should be mandatory to rule out any recurrence in future. We conclude that conservative management by enucleation and chemical cauterization of bony cavity using carnoy's solution and placement of CGF along with aotogenous bone graft mixed with remaining RBC in the dead space which induce osteoblastic activity, which in turn useful for future rehabilitation for placing dental implants or denture, so it is the treatment of choice for extensive case of KCOT's,

\section{Acknowledgement}

1) Dr.A.S.Ganesan, Honourable Chancellor, Vinayaga mission University, Kilpauk, Chennai, for his kind support, \&

2) Prof.Dr.P.Anusuya, Dean, Arupadaivedu Medical College \& Hospital, Puducherry, for his eminent guidance,

3) Dr.T. Ramesh, Managing Director, Adiparasakthi Dental College \& Hospital, Melmaruvathur. 


\section{References}

[1]. Philipsen HP. Om keratocystedr (Kolesteratomer) and kaeberne. Tandlaegebladet 1956; 60:963-71

[2]. Voorsmit R.A., Stoelinga P.J., van Haelst U.J. The management of keratocysts. J. Maxillofac. Surg. 1981;9:228

[3]. Choukroun J, Diss A, Simonpieri A, Girard MO, Schoeffler C, Dohan SL, et al. Platelet-rich fibrin (PRF): A second-generation platelet concentrate. Part V: Histologic evaluations of PRF effects on bone allograft maturation in sinus lift. Oral Surg Oral Med Oral Pathol Oral Radiol Endod. 2006;101:299-303.

[4]. Sacco L. Lecture, International academy of implant prosthesis and osteoconnection, 2006. 12. 4.

[5]. Sohn DS, Moon JW, Moon YS, Park JS, Jung HS. The use of concentrated growth factors (CGF) for sinus augmentation. Journal of Oral Implant (Japan) 2009;38:25-35.

[6]. Bramley PA. Treatment of cysts of the jaws. Proc R Soc Med. 1971 May;64(5):547-550.

[7]. Shear M, Altini M. Odontogenic and non-odontogenic cysts of the jaws. J Dent Assoc S Afr. 1983 Sep;38(9):555-60, 562-4

[8]. 8.Bradley P.F., Fisher A.D. The cryosurgery of bone, an experimental and clinical assessment. Br. J. Oral Surg. 1975 Nov;13(2):111-27.

[9]. 9. Massimo, Luigi, Edoardo; International Academy of Implant Prosthesis and Ossteoconnections, Univ. of Sassari, Italy : 2013 